consensus or ideology, and fake-news sustains and consolidates prior beliefs. These powerful forces are generated by political, economic and religious processes and realities, which must be factored into any public communications and determine their every detail

3. Regulatory, bureaucratic and many scientific communications fail to take this complexity into account in both the presentation of evidence and in its defence

4. Exploiting some of the wisdom of Aristotle's Art of Rhetoric, and using some of the insights of modern sociology, psychology and neuroscience, specific reforms in medical and scientific communications and public engagement are proposed. The resurrection we need is that of genuine social discourse.

Conclusions With few exceptions, scientific, regulatory and bureaucratic communications in medicine, have fallen far behind the dynamism and diversity of modern popular culture, especially digital media. Scepticism about vaccination (and climate change, of course); the embracing of alternative and charismatic practices and therapies; the dismissive attitudes to causality and evidence; the vast failures in adherence; the seemingly irresistible trade in fake and substandard medicines; the opioid crisis; infant and maternal mortality; medical and medication errors and the worldwide abuse of antibiotics hardly any of these are either countered with the skill, energy, creativity and seductiveness of their promotion, or equal in response to the intensity of their entrenchment.

Proposals for debate and change radical community engagement; nurturing of ambassadors for science; prioritisation and ranking of core issues; creation of evidence stories; revolution in use of language; exploration of the causes of scepticism; dynamic exploitation of digital devices and channels.

\section{ROLE OF SCIENTIFIC SOCIETIES IN GUIDELINES DEVELOPMENT. THE CASE STUDY OF THE EUROPEAN SOCIETY OF CLINICAL MICROBIOLOGY AND INFECTIOUS DISEASES}

\begin{abstract}
1,2 Luigia Scudeller, ${ }^{3,4}$ Lorenzo Guglielmetti, ${ }^{5,6}$ Khamet Hussein, ${ }^{7}$ Benedikt Huttner, ${ }^{8}$ Amel Letaief, ${ }^{9}$ Sarah Tschudin-Sutter, ${ }^{10}$ Francois Vandenesch, ${ }^{11,12}$ Jesus Rodriguez Bano, 13,14,15Evelina Tacconelli. 'Fondazione IRCCS Policlinic San Matteo, PAVIA (PV), Italy; ${ }^{2}$ ESCMID Guidelines Director, Basel, Switzerland; ${ }^{3} A P-H P$, Laboratoire de BactériologieHygiène, Centre National de Référence des Mycobactéries et de la Résistance des Mycobactéries aux Antituberculeux, Hôpitaux Universitaires Pitié Salpêtrière-Charles Foix, Paris, France; ${ }^{4}$ Sorbonne Université, Centre d'Immunologie et des Maladies Infectieuses (CIMI; INSERM U1135/UMRS CR7/CNRS ERL 8255), Bactériologie, Faculté de Médecine Sorbonne Université, Paris, France; ${ }^{5}$ Infectious Diseases Institute. Rambam Health Care Campus, Haifa, Israel; ${ }^{6}$ The Ruth and Bruce Rappaport Faculty of Medicine, Technion, Israel Institute of Technology, Haifa, Israel; 'Division of Infectious Diseases, Geneva University Hospitals and Faculty of Medicine, University of Geneva 4, Rue Gabrielle Perret-Gentil, CH1211, Geneva, Switzerland; ${ }^{8}$ Infectious Diseases Department, Hôpital Farhat Hached, Université de Sousse, Sousse, Tunisia; ${ }^{9}$ Division of Infectious Diseases and Hospital Epidemiology Basel University Hospital, Basel, Switzerland; ${ }^{10}$ Centre International de Recherche en Infectiologie, University of Lyon, Lyon, France; ${ }^{11}$ Infectious Diseases Division at Hospital Universitario Virgen Macarena, Universidad de Sevilla, Sevilla, Spain; ${ }^{12}$ ESCMID president, Basel, Switzerland; ${ }^{13}$ Comprehensive Center of Infectious Diseases, Tübingen University, Tubingen, Germany; ${ }^{14}$ of Infectious Diseases Clinic, University of Verona, Verona, Italy; ${ }^{15}$ ESCMID Guidelines Officer, Basel, Switzerland
\end{abstract}

\subsection{6/bmjebm-2019-EBMLive.84}

Objectives Great challenges lay ahead of scientific societies as providers of guidance documents. On one hand, they are under increased scrutiny to develop trustworthy and useful guidelines; it has even been advocated that professional societies should abstain from authoring guidelines and disease definition statements. On the other hand, scientific societies are requested by clinicians, policy makers and patients alike, to be more efficient in producing guidance documents on a large number of topics, often in the absence of strong evidence upon which to base recommendations.

The European Society of Clinical Microbiology and Infectious Diseases (ESCMID) is revising its programme for guideline development to meet the huge challenges in how guidance documents are perceived (trustworthy guidelines) and developed (useful guidelines). We discuss here lessons learnt and plans for the future.

Method ESCMID (founded in 1983) is Europe's leading society in clinical microbiology and infectious diseases, with 30,000 members from all European countries and all continents, 30 study groups, and many national and international affiliated institutions. ESCMID is part of the Guidelines International Network. In the past few years, many guidance documents were produced under the ESCMID name; however, ESCMID's main role was one of networking, general oversight and peer review. Staff was limited to 0.5 full-time equivalents, with only an administrative role. The role of the guideline director was liaison between potential drafting groups and the Executive Committee, general oversight of conflict of interest, coordination with other scientific societies in cooperative projects. Industry-funding of guidelines has never been accepted. Since 2017, we reviewed our procedures and developed a strategy to improve quality, rigour and transparency of guidelines issued by our society.

Results The three main challenges we identified are: conflicts of interest of members and society, leading to potentially biased guidance; lack of knowledge of methods in guideline development even among experienced members (with resistance to change as an additional consequence); reliance on commitment of individual professionals, leading to risk of poor sustainability. In response, we have devised a multifaceted intervention. We are involving, via open calls, a larger number of professionals, to create a network (not limited to Europe) able to manage, control, monitor and oversee the many practical, scientific and ethical issues involving clinical guidance documents. We created different bodies/groups, to ensure reciprocal oversight on different activities. We involved stakeholders globally, to select priority topics, with appropriate funding. We are organizing online and face-to-face training on guideline development methods and implementing tools to assist guideline development groups. Most importantly, we are updating our policy and strategy for conflict of interest control and management.

Conclusions The science and art of guidance production is complex and important in these times of information overload, resource constraints in health care and shrinking research budgets. We highlighted many issues awaiting guideline production in our fields of medicine and are working to address them. The pillars of our strategy are to: select cutting-edge guideline topics, apply an independent and rigorous methodology; ensure a global perspective; appoint members to guideline-related tasks based on merit; involve young ESCMID members to ensure sustainability of the model; strictly monitor conflict of interest declarations.

We here call for external, independent, advisors to participate in our programme.

We are confident that, with the contribution of excellent professionals (ESCMID and not), and with the balances and 
checks in our new structure, we will meet the high challenges we are facing, and that professional societies can still play a role in producing trustworthy and useful clinical guidance documents.

\section{TAKING ACCOUNT OF TUMOUR HETEROGENEITY IN SYSTEMATIC REVIEWS}

Shona Lang, Stephanie Swift, Steve Duffy, Jos Kleijnen. Kleijnen Systematic Reviews Ltd, York, UK

\subsection{6/bmjebm-2019-EBMLive.85}

Objectives Precision medicine requires an in-depth understanding of cancer genomics and a rigorous approach to tumour tissue evaluation. Accounting for tumour heterogeneity, which is a feature of most cancers, is essential when assessing somatic mutations. Genomic instability produces multiple tumour clones within a given tumour tissue. Each clone has different characteristics (e.g. histology, growth, genetic mutations) and ultimately, can have different clinical outcomes. The challenge in oncology is to work out which clone is the greatest threat to life and target treatment appropriately. Current approaches to biomarker testing focus on interpatient heterogeneity but not intra-tumour heterogeneity.

We aim to evaluate how somatic mutation studies report and validate tumour tissue samples and whether they consider tumour heterogeneity. Secondly, we aim to search the literature to identify existing guidance on tumour heterogeneity.

Method We used a set of nine studies (12 datasets) taken from a recent systematic review on the prevalence of somatic gene mutations in cancer. We assessed whether the authors of the studies reported on:

1. Tissue sampling:

i. the clarity of reporting for sampling and pathology methods

ii. whether the pathology of the sample or the patient is presented.

2. Tumour heterogeneity or clonality:

i. the use of multiple samples from the same patient;

ii. the investigation of clonality or heterogeneity.

3. Tumour content (purity):

i. the use of microdissection;

ii. the reporting of the tumour content of the sample.

We searched MEDLINE, Pubmed and Embase and seven guideline- and systematic review-specific databases from inception to January 2019 to identify guidelines reporting on tumour heterogeneity.

Results Seven (58\%) datasets did not report sufficient information with regards to tumour sampling and pathology to confirm that the tumour sample represented the patient's pathology. The use of this information in a systematic review would introduce uncertainty.

None of the datasets reported multiple prevalence results per sample. The use of this finding in a review would illustrate an evidence gap that tumour heterogeneity was not investigated only patient heterogeneity.

Ten datasets $(83 \%)$ reported measuring the tumour content of the sample; seven (58\%) reported that the tumour content could be as low as $10 \%$. The use of this finding in a review would illustrate that the samples are at risk of contamination and the results cannot be considered reliable.
Results for searching of guidelines will be presented at the conference.

Conclusions Systematic reviewers need to be much more cautious about the use of patient derived tissue samples. It cannot be assumed that a tumour sample replicates the pathology of the patient, or that somatic tumour samples are homogeneous for a given biomarker or mutation. Systematic reviewers need to assess whether a) the study reports sufficient information to confirm that the sample reflects the pathology of interest b) the sample is free of normal, benign or other contaminant cells c) tumour heterogeneity has been considered. The assessment of tumour samples and tumour heterogeneity is important for systematic reviews of epidemiology, diagnostic and prognostic studies.

\section{HOW DO WE KNOW THAT WE KNOW SOMETHING?}

${ }^{1,2}$ Carlos José Fajardo Flores, ${ }^{3}$ Jackeline Alger. 'Universidad Nacional Autónoma de Honduras, Tegucigalpa, Honduras; ${ }^{2}$ Centro de Cáncer Emma Romero de Callejas, Tegucigalpa, Honduras; ${ }^{3}$ Universidad Nacional Autónoma de Honduras, Tegucigalpa, Honduras

\subsection{6/bmjebm-2019-EBMLive.86}

In high income countries, this question may be substituted by another one that it's easier to answer: How many randomized controlled trials have been done over this topic? In Honduras, Central America, this question is answered very differently. Medical practice in LMIC continues to be empirical and anecdotic and in the best-case scenario, decisions are taken based on research made abroad under distinct settings. Dealing with endless challenges, our health system fails to inform operational, implementation and health system research decisions. Barriers that limit quality research go from untrained health workers to lack of funding to come up with more complex research designs. Descriptive studies represent an overwhelming majority of published studies but due to their nature, their impact falls short to inform health decisions that benefit those that need it the most. So, are we posing quality research studies that answer our most important clinical questions? The EBM manifesto enlists the most important challenges that need to be addressed. Increase the systematic use of evidence is one of them. An estimated $97 \%$ of research in the world seeks to produce new knowledge, only the remaining 3\% seeks to implement existing knowledge. Implementation Research (IR) is a discipline that aims to uptake interventions that have been proven effective under controlled settings and turn them into routine practices that benefit a wider population. Let's consider EBM as an effective intervention to enhance quality research practice, then why in LMIC an EBM culture has not been implemented? What factors have diminished its impact? IR might be an important tool to uptake the implementation of EBM in a poor developed research context. Pose research questions that arise in the communities, in real not controlled settings, that try to solve day-to-day problems can make the research process a less cumbersome enterprise. In other words, more practical questions that are closer to reality can boost a research practice that motivates a scientific community that watches research more as a luxury than as a necessity. A powerful way to motivate a scientific community to uptake 